\title{
Morfologia polínica de espécies de Brunfelsia L. (Solanaceae) ocorrentes no Estado do Rio de Janeiro ${ }^{1}$
}

\author{
CARLA P.R. BATISTA-FRANKLIM ${ }^{2,3}$ e VANIA GONÇALVES-ESTEVES ${ }^{2}$
}

(recebido: 20 de abril de 2000; aceito: 21 de fevereiro de 2002)

\begin{abstract}
Pollinic morphology of species of Brunfelsia L. (Solanaceae) occuring in state of Rio de Janeiro) - This work presents an analysis of the pollen grains of six taxa of the genera Brunfelsia: B. bonodora (Vell.) J.F. Macbr., B. brasiliensis (Spreng.) L.B. Sm. \& Downs var. brasiliensis, B. brasiliensis subsp. macrocalyx (Dusén) Plowman, B. hydrangeiformis subsp. capitata (Benth.) Plowman, B. latifolia (Pohl) Benth. e B. uniflora D. Don. The pollen grains were treated with ACLAC $60 \%$ (except the pollen grains of $B$. latifolia that were submitted to acetolysis). Afterwards, they were measured and photographed by light microscopy and SEM. The analysis under SEM was used to check the sculpturing of the exine. It was observed that the pollen grains are medium or large; isopolar; suboblate or oblate spheroidal; the amb is subcircular, square to pentagonal; the polar area is very small to large; 3-5-colporate; the sexine is variably rugulated, but best visualized in SEM.

RESUMO - (Morfologia polínica de espécies de Brunfelsia L. (Solanaceae) ocorrentes no Estado do Rio de Janeiro) - Neste trabalho foram analisados os grãos de pólen de seis táxons do gênero Brunfelsia: B. bonodora (Vell.) J.F. Macbr., B. brasiliensis (Spreng.) L.B. Sm. \& Downs var. brasiliensis, B. brasiliensis subsp. macrocalyx (Dusén) Plowman, B. hydrangeiformis subsp. capitata (Benth.) Plowman, B. latifolia (Pohl) Benth. e B. uniflora D. Don. Os grãos de pólen foram tratados com ACLAC $60 \%$ (exceto os de B. latifolia, que sofreram o processo da acetólise). Os grãos de pólen foram, posteriormente, mensurados, descritos, foto e eletromicrografados. A análise sob MEV foi utilizada visando a elucidação de dúvidas sobre a ornamentação da exina. Constatou-se que os grãos de pólen são médios ou grandes; isopolares; suboblatos ou oblato-esferoidais; âmbito subcircular, quadrangular a pentagonal; área polar de muito pequena a grande; 3-5-colporados; sexina variavelmente rugulada porém, melhor visualizada sob MEV.
\end{abstract}

Key words - Pollinic morphology, Brunfelsia, Solanaceae

\section{Introdução}

D’Arcy (1991), numa revisão biogeográfica mais recente de Solanaceae, aceitou três subfamílias: Solanoideae (com oito tribos), Nolanoideae (com uma tribo) e Cestroideae (com cinco tribos, dentre as quais está Salpiglossideae com dez gêneros). Brunfelsia L. é um dos gêneros subordinados à essa tribo. Carvalho (1997) divulgou uma lista preliminar das espécies de Solanaceae ocorrentes no Estado do Rio de Janeiro e, segundo a autora, Cestroideae está representada neste estado por sete gêneros, dentre eles Brunfelsia com nove táxons.

Sobre a palinologia de Solanaceae há poucos registros de publicações com espécies brasileiras podendo-se ressaltar os trabalhos de Salgado-Labouriau (1973) e Wiebke \& Wiebke (1974). Dados palinológicos sobre essa família são poucos e aparecem,

\footnotetext{
1. Parte da dissertação de mestrado da primeira autora, Pós-Graduação em Botânica, Museu Nacional/UFRJ.

2. Museu Nacional/Universidade Federal do Rio de Janeiro, Departamento de Botânica, Quinta da Boa Vista, São Cristóvão, 20940-040 Rio de Janeiro, RJ, Brasil.

3._Autor para correspondência: vesteves@acd.ufrj.br
}

freqüentemente, em floras polínicas de diversas regiões do mundo e em trabalhos apresentados nos Simpósios Internacionais de Biologia e Taxonomia de Solanáceas.

Poucos autores estudaram os grãos de pólen de Brunfelsia e dentre eles, é possível citar os trabalhos de Rao \& Leong (1974), que analisaram a morfologia polínica de algumas plantas tropicais; Miranda et al. (1978), que estudaram os grãos de pólen de espécies pertencentes a várias famílias encontradas no Ceará; Gentry Jr. (1979), que descreveu a morfologia polínica de cerca de 130 espécies de Salpiglossidae (Solanaceae) e Batista (1999), que estudou a palinologia de 15 táxons distribuídos em cinco gêneros de Solanaceae encontrados no Estado do Rio de Janeiro.

O presente estudo baseou-se na revisão biogeográfica feita por D’Arcy (1991) e resultou na caracterização morfopolínica de seis táxons: B. bonodora (Vell.) J.F. Macbr., B. brasiliensis (Spreng.) L.B. Sm. \& Downs var. brasiliensis, B. brasiliensis subsp. macrocalyx (Dusén) Plowman, B. hydrangeiformis subsp. capitata (Benth.) Plowman, B. latifolia (Pohl) Benth. e B. uniflora (Pohl) D. Don. Devido à falta de material polínico nos herbários consultados, não puderam ser analisados os grãos de pólen de B. hydrangeiformis (Pohl) Benth. subsp. 
hydrangeiformis, B. pauciflora (Cham. \& Schltdl.) Benth. subsp. pauciflora e B. pilosa Plowman.

Com os resultados obtidos buscou-se caracterizar, palinologicamente, os táxons acima mencionados, assim como contribuir para a formação de um catálogo polínico da flora do Estado do Rio de Janeiro.

\section{Material e métodos}

O material polínico foi retirado de exsicatas depositadas nos herbários: Alberto Castellanos - FEEMA (GUA), Instituto de Pesquisas Jardim Botânico do Rio de Janeiro (R) e Museu Nacional (RB). Siglas de acordo com Holmgren et al. 1990.

Para cada táxon, determinou-se um espécime que foi considerado padrão (assinalado abaixo por asterisco) procurando-se estudar, sempre que possível, outros espécimes de cada táxon (um máximo de três), então denominados materiais de comparação. Para os táxons B. bonodora e $B$. hydrangeiformis subsp. capitata não foram encontrados materiais para comparação.

Brunfelsia bonodora: BRASIL: RIO DE JANEIRO: Barra Mansa, 3-XII-1960, A.P. Duarte 5.477 (RB*). Brunfelsia brasiliensis var. brasiliensis: BRASIL: RIO DE JANEIRO: Petrópolis, IX-1943, O.C. Goés \& D. Constantino 543 (RB); idem, Retiro, XII-1943, O.C. Goés \& D. Constantino 710 (RB*); Rio de Janeiro, 18-I-1931, Pico da Tijuca, A.C. Brade 10.583 (R). Brunfelsia brasiliensis subsp. macrocalyx: BRASIL: RIO DE JANEIRO: Itatiaia, Parque Nacional do Itatiaia, Km 8, 11-X-1977, P.J.M. Maas \& G. Martinelli 3.209 (RB); idem, entre Marombas e Macieiras, Parque Nacional do Itatiaia, $\pm 1.700 \mathrm{~m}, 17-\mathrm{VIII}-1969$, D. Sucre $5.806(\mathrm{RB} *)$; Teresópolis, Posse, 10-II-1968, D. Sucre 2.326 \& P.I.S. Braga 169 (RB). Brunfelsia hydrangeiformis subsp. capitata: BRASIL: Rio DE JANEIRO: Rio de Janeiro, Tijuca, Pico do Papagaio, 29-XI-1928, A. Ducke (RB*). Brunfelsia latifolia: BRASIL: Rio DE JANeIro: Maricá, Praia de Itaipuaçu, 11-IV-1986, D. Araújo 7.372 (GUA); idem, Restinga de Itaipuaçu, 21-IX-1992, V. Esteves \& R. Esteves 910 (R); Rio de Janeiro, Restinga de Jacarepaguá, 27-XI-1969, D. Sucre et al. 6.428 (RB*). Brunfelsia uniflora: BRASIL: RIO DE JANEIRO: Maricá, Itaipuaçu, Pico Alto Moirão, 26-VIII-1981, R. Andreata 100 \& H.C. Lima 1.637 (RB); Rio de Janeiro, Cantagalo, próximo a Euclidelândia, 15-XII-1967, L. Emygdio 2.600 ( $\left.\mathrm{R}^{*}\right)$; idem, Restinga da Gávea, 28-IX-1949, s.n. (RB 75.565).

Para a análise sob microscopia de luz foi utilizado o método da acetólise láctica a 60\% (Raynal \& Raynal 1971) por serem os grãos de pólen, na maioria, muito frágeis. Apenas os grãos de pólen de B. latifolia resistiram ao tratamento acetolítico de Erdtman (1952). Para a montagem das lâminas, foram colocadas esferas de massa de modelagem (ca. $1 \mathrm{~mm}$ de diâmetro) entre a lâmina e a lamínula para evitar o amassamento dos grãos de pólen com parede frágil (Reitsma 1969). Para melhor visualização da forma e do tipo de abertura, alguns grãos de pólen foram tratados segundo o método de Wodehouse (1935). Os grãos de pólen foram medidos e fotomicrografados até três dias após sua preparação (Wanderley \& Melhem 1991).

Para a obtenção das eletromicrografias, 2 a 3 anteras foram maceradas, liberando os grãos de pólen (não acetolisados) diretamente sobre um suporte metálico previamente recoberto por fita adesiva de carbono dupla face. O material sobre o suporte foi metalizado em ouro puro por cerca de três minutos e analisado, posteriormente, em aparelho Zeiss DSM 960.

Do material padrão foram medidos em 25 grãos de pólen, o diâmetro equatorial em vista polar (DEVP), e os diâmetros polar (DP) e equatorial (DE) em vista equatorial. Foram realizados os tratamentos estatísticos calculando-se a média aritmética $(\overline{\mathrm{x}})$, o desvio padrão da amostra (s), o desvio padrão da média $\left(\mathrm{s}_{\overline{\mathrm{x}}}\right)$ coeficiente de variabilidade (CV\%) e o intervalo de confiança a 95\% (IC 95\%) (Vieira 1981). Para os demais caracteres como o lado do apocolpo (LA), as aberturas e as camadas da exina, foram calculadas as médias aritméticas de 10 medidas, o mesmo ocorrendo para as medidas dos diâmetros dos grãos de pólen dos materiais de comparação.

As descrições tiveram como base as observações realizadas sob microscópio de luz (sensu Erdtman 1952); a terminologia adotada foi a de Barth \& Melhem (1988) e a de Punt et al. (1999).

\section{Resultados}

As espécies de Brunfelsia aqui estudadas (figuras 1-37) apresentaram grãos de pólen médios, grandes apenas em $B$. hydrangeiformis subsp. capitata (tabela 1), isopolares; oblato-esferoidais (suboblatos apenas em B. uniflora); âmbito subcircular (circular, quadrangular a pentagonal em B. brasiliensis var. brasiliensis e $B$. brasiliensis subsp. macrocalyx); área polar muito pequena, pequena ou grande (tabelas 2, 3), 3-5-colporados; sexina variavelmente rugulada (tabela 3 ).

Os colpos variaram de curtos a longos ou muito longos (tabelas 3,4) apenas em B. uniflora, endoabertura lolongada na maioria dos táxons (tabela 4), lalongada em B. bonodora e B. uniflora apesar de ser de difícil visualização em $B$. bonodora (figura 6), B. brasiliensis var. brasiliensis e $B$. brasiliensis subsp. macrocalyx. O número de aberturas variou de 3 a 5 na maioria das espécies (figuras $8,9,25,26$ ), enquanto $B$. uniflora não apresentou esta variação (figura 34), possuindo, apenas 3 aberturas (tabela 3). 

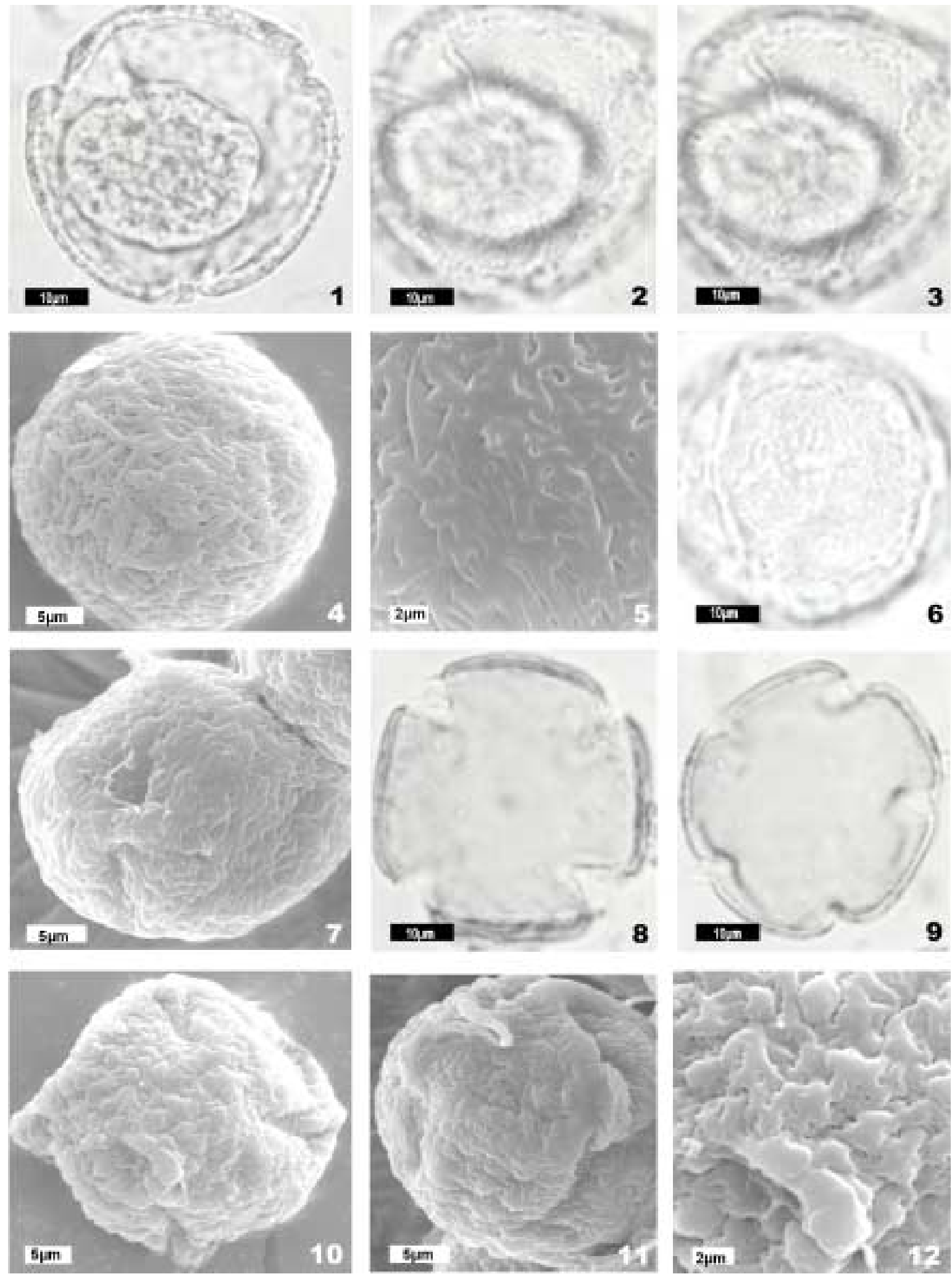

Figuras 1-12. Fotomicrografias e eletromicrografias dos grãos de pólen de espécies de Brunfelsia. 1-7. B. bonodora. 1. Corte óptico em vista polar; 2-3. Análise de L.O. em vista polar; 4. Vista polar (MEV); 5. Detalhe da superfície em vista polar (MEV); 6. Vista equatorial, 7. Superfície e abertura em vista equatorial (MEV). 8-12. B. brasiliensis var. brasiliensis. 8. Corte óptico de grão de pólen 4-colporado em vista polar; 9. Corte óptico de grão de pólen 5-colporado em vista polar; 10. Vista polar (MEV); 11. Abertura em vista equatorial; 12. Detalhe da superfície no mesocolpo em vista equatorial (MEV). 

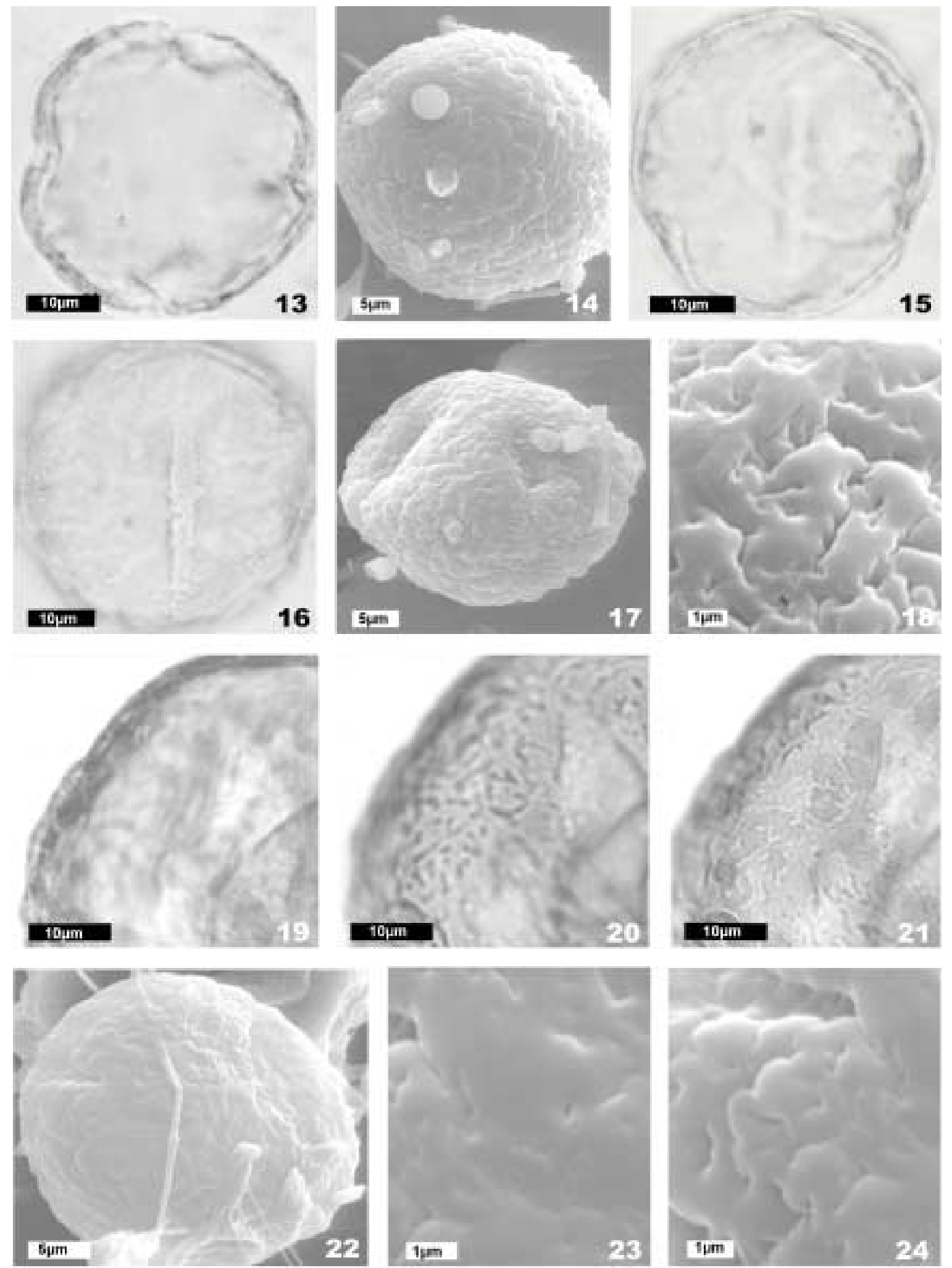

Figuras 13-24. Fotomicrografias e eletromicrografias dos grãos de pólen de espécies de Brunfelsia. 13-18. B. brasiliensis subsp. macrocalyx. 13. Corte óptico em vista polar; 14. Vista polar (MEV); 15. Corte óptico em vista equatorial; 16. Abertura em vista equatorial; 17. Vista lateral (MEV); 18. Detalhe da superfície em vista equatorial (MEV). 19-24. B. hydrangeiformis subsp. capitata. 19. Corte óptico em vista equatorial; 20-21. Análise de L.O. em vista equatorial; 22. Vista polar (MEV); 23. Detalhe da superfície em vista polar (MEV); 24. Detalhe da superfície e da membrana ornamentada do colpo em vista polar. 

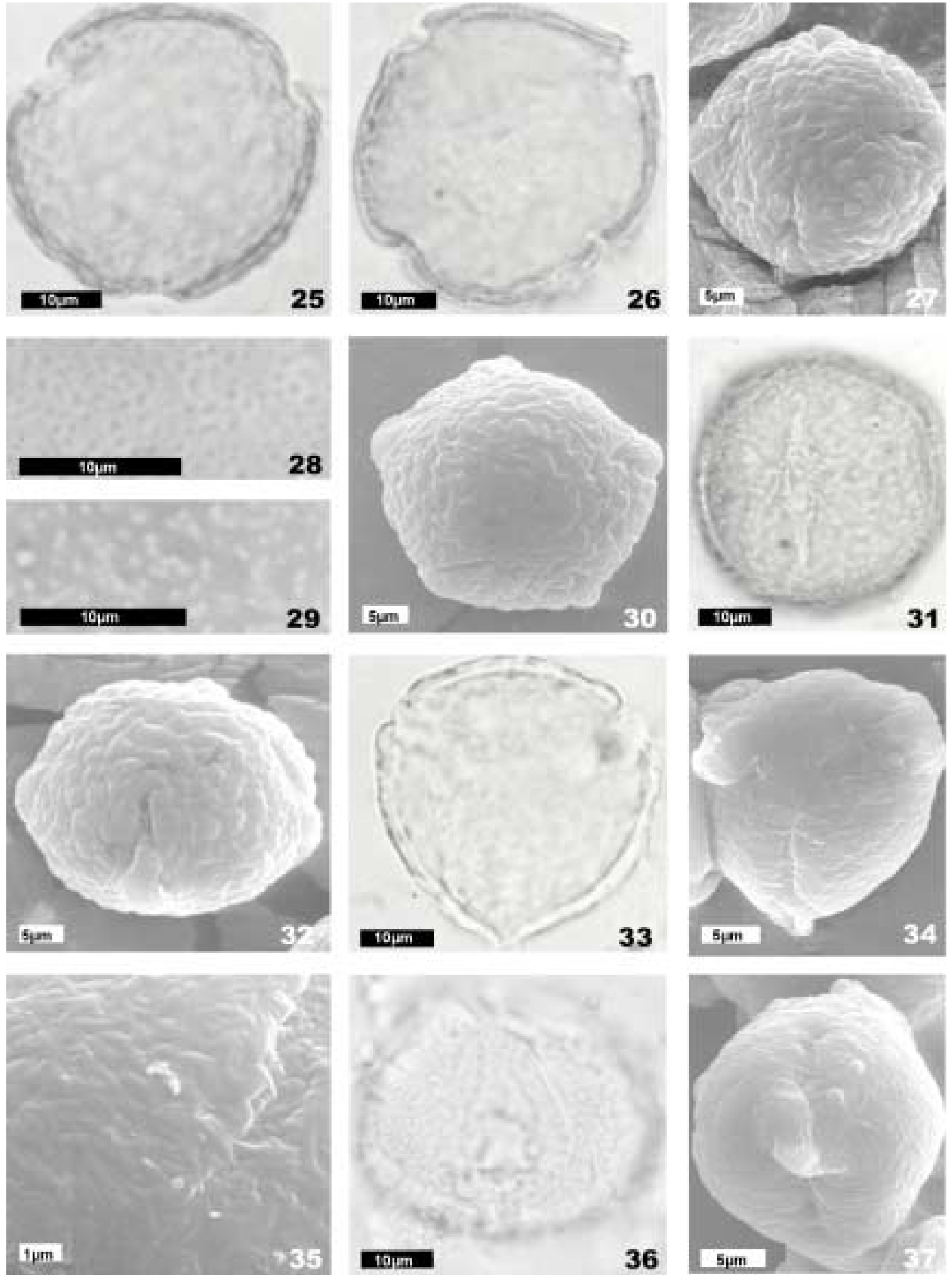

Figuras 25-37. Fotomicrografias e eletromicrografias dos grãos de pólen de espécies de Brunfelsia. 25-33. Grãos de pólen de B. latifolia. 25. Corte óptico de grão de pólen 3-colporado em vista polar; 26. Corte óptico de grão de pólen 4-colporado em vista polar; 27. Grão de pólen 4-colporado em vista polar (MEV), 28-29. Análise de L.O.; 30. Grão de pólen 5-colporado em vista polar (MEV); 31. Superfície e abertura em vista equatorial; 32. Vista equatorial (MEV). 33-37. B. uniflora. 33. Corte óptico em vista polar; 34. Vista polar (MEV); 35. Detalhe da superfície e da membrana ornamentada do colpo em vista polar (MEV); 36. Superfície e abertura em vista equatorial; 37. Vista equatorial (MEV). 
Tabela 1. Medidas $(\mu \mathrm{m})$ dos grãos de pólen de táxons de Brunfelsia, em vista equatorial $(\mathrm{n}=25) . \overline{\mathrm{x}}=$ média aritmética; $\mathrm{s}_{\overline{\mathrm{x}}}=$ desvio padrão da média; $\mathrm{s}=$ desvio padrão da amostra; $\mathrm{CV} \%$ = coeficiente de variabilidade.

\begin{tabular}{|c|c|c|c|c|c|c|c|c|c|c|}
\hline \multirow[t]{2}{*}{ Táxons } & \multicolumn{5}{|c|}{ Diâmetro polar } & \multicolumn{5}{|c|}{ Diâmetro equatorial } \\
\hline & $\begin{array}{l}\text { Faixa de } \\
\text { variação }\end{array}$ & $\overline{\mathrm{x}} \pm \mathrm{s}_{\overline{\mathrm{x}}}$ & $\mathrm{s}$ & $\mathrm{CV} \%$ & $\begin{array}{l}\text { Intervalo de } \\
\text { confiança }\end{array}$ & $\begin{array}{l}\text { Faixa de } \\
\text { variação }\end{array}$ & $\overline{\mathrm{x}} \pm \mathrm{s}_{\overline{\mathrm{x}}}$ & $\mathrm{s}$ & CV\% & $\begin{array}{l}\text { Intervalo de } \\
\text { confiança }\end{array}$ \\
\hline B. bonodora & $46,0-50,0$ & $47,9 \pm 0,3$ & 1,3 & 2,7 & $47,3-48,5$ & $45,0-50,0$ & $47,5 \pm 0,4$ & 1,9 & 4,0 & $46,7-48,3$ \\
\hline $\begin{array}{l}\text { B. brasiliensis } \\
\text { var. brasiliensis }\end{array}$ & $40,0-43,7$ & $41,5 \pm 0,3$ & 1,2 & 2,9 & $40,9-42,1$ & $42,5-43,7$ & $42,8 \pm 0,1$ & 0,5 & 1,2 & $42,6-43,0$ \\
\hline $\begin{array}{l}\text { B. brasiliensis } \\
\text { subsp. macrocalyx }\end{array}$ & $45,0-47,5$ & $46,7 \pm 0,2$ & 0,9 & 1,9 & $46,3-47,1$ & $46,2-47,5$ & $46,9 \pm 0,1$ & 0,6 & 1,3 & $46,7-47,1$ \\
\hline $\begin{array}{l}\text { B. hydrangeiformis } \\
\text { subsp. capitata }\end{array}$ & $56,2-62,5$ & $59,2 \pm 0,3$ & 1,5 & 2,5 & $58,6-59,8$ & $60,0-70,0$ & $67,1 \pm 0,6$ & 2,9 & 4,3 & $65,9-68,3$ \\
\hline B. latifolia & $40,0-45,0$ & $42,8 \pm 0,3$ & 1,3 & 3,0 & $42,2-43,4$ & $42,2-43,4$ & $42,8 \pm 0,3$ & 1,4 & 3,0 & $42,2-43,4$ \\
\hline B. uniflora & $36,2-40,0$ & $38,1 \pm 0,2$ & 1,2 & 3,1 & $37,7-38,5$ & $42,5-47,5$ & $44,7 \pm 0,4$ & 2,0 & 4,5 & $43,9-45,5$ \\
\hline
\end{tabular}

Sob MEV os colpos apresentaram membrana ornamentada e margem não diferenciada (figuras 7 , 17, 37).

A exina apresentou-se rugulada, com rúgulas conspícuas ou menos conspícuas (tabela 3) como, respectivamente, em $B$. brasiliensis var. brasiliensis (figura 12) e B. uniflora (figuras 35, 36), teto tênue e ondulado; sexina ligeiramente mais espessa ou com espessura igual à da nexina, em B. uniflora (tabela 4); nexina 1 mais espessa e nexina 2 mensurável apenas em B. uniflora. Essa última apresentou-se como uma camada descontínua. As eletromicrografias mostraram que a sexina é variavelmente rugulada e perfurada. $B$. bonodora apresentou, em regiões do mesocolpo, rúgulas alongadas semelhantes a estrias (figura 7). As rúgulas são muito conspícuas em $B$. brasiliensis var. brasiliensis (figura 12) e em B. brasiliensis subsp. macrocalyx (figura 17) apenas no mesocolpo. Em B. latifolia, esta região apresentou ornamentação da sexina semelhante a ínsulas (figura 32) e nos pólos a sexina torna-se rugulada (figuras 27, 31).

Em B. brasiliensis var. brasiliensis observou-se que $75 \%$ dos grãos de pólen eram médios $(\overline{\mathrm{x}}=42,8 \mu \mathrm{m})$, prolato-esferoidais, 4-colporados e $25 \%$ eram grandes $(\overline{\mathrm{x}}=52,5 \mu \mathrm{m})$, tendendo à forma esferoidal $\mathrm{e}$ 5-colporados.

Constatou-se que os grãos de pólen dos espécimes de comparação estudados mantiveram constantes a forma (tabela 5), a ornamentação da exina e a variação no número de aberturas quando comparados com os respectivos materiais padrões, exceto em $B$. uniflora cujos materiais de comparação apresentaram forma diferente.

Os grãos de pólen de $B$. bonodora, B. brasiliensis var. brasiliensis e $B$. brasiliensis subsp. macrocalyx foram aqueles que apresentaram a maior dificuldade na distinção do tipo de abertura. Essa dificuldade na interpretação pode ser superada quando foram analisados grãos de pólen corados com safranina (para microscopia de luz) e estudados sob MEV.

Tabela 2. Medidas $(\mu \mathrm{m})$ dos grãos de pólen de táxons de Brunfelsia, em vista polar: diâmetro equatorial $(\mathrm{n}=25)$; lado do apocolpo (LA) e índice da área polar (IAP) $(\mathrm{n}=10) . \overline{\mathrm{x}}=$ média aritmética; $\mathrm{s}_{\overline{\mathrm{x}}}=$ desvio padrão da média; $\mathrm{s}=$ desvio padrão da amostra; $\mathrm{CV} \%$ = coeficiente de variabilidade.

\begin{tabular}{lccccccc}
\hline Táxons & \multicolumn{5}{c}{ Diâmetro equatorial } & LA & IAP \\
\cline { 2 - 6 } & Faixa de variação & $\overline{\mathrm{x}} \pm \mathrm{s}_{\overline{\mathrm{x}}}$ & $\mathrm{s}$ & CV\% & Intervalo de confiança & $\overline{\mathrm{x}}$ & \\
\hline B. bonodora & $45,0-50,0$ & $47,5 \pm 0,4$ & 1,9 & 4,0 & $46,7-48,3$ & 31,3 & 0,66 \\
B. brasiliensis var. brasiliensis & $45,0-50,0$ & $47,1 \pm 0,3$ & 1,7 & 3,6 & $46,5-47,7$ & 22,5 & 0,48 \\
B. brasiliensis subsp. macrocalyx & $42,5-47,5$ & $45,0 \pm 0,4$ & 1,8 & 4,0 & $44,2-45,8$ & 22,0 & 0,49 \\
B. hydrangeiformis subsp. capitata & $57,5-65,0$ & $61,2 \pm 0,5$ & 2,5 & 4,1 & $60,2-62,2$ & 35,4 & 0,58 \\
B. latifolia & $43,7-46,2$ & $45,2 \pm 0,2$ & 1,0 & 2,2 & $44,8-45,6$ & 11,5 & 0,25 \\
B. uniflora & $40,0-45,0$ & $42,2 \pm 0,3$ & 1,5 & 3,6 & $41,6-42,8$ & 10,6 & 0,25 \\
\hline
\end{tabular}


Tabela 3. Caracterização morfológica dos grãos de pólen de táxons de Brunfelsia. P/E = relação entre a média do diâmetro polar $(\mathrm{P})$ e a média do diâmetro equatorial $(\mathrm{E})$.

\begin{tabular}{lccccc}
\hline Táxons & N. de aberturas & Colpo & Área polar & P/E & Rúgulas \\
\hline B. bonodora & $3(90 \%)-4(10 \%)$ & curto & grande & 0,96 & conspícuas \\
B. brasiliensis var. brasiliensis & $4(75 \%)-5(25 \%)$ & longo & pequena & 0,97 & pouco conspícuas \\
B. brasiliensis subsp. macrocalyx & $3(12 \%)-4(85 \%)-5(3 \%)$ & longo & pequena & 0,99 & conspícuas \\
B. hydrangeiformis subsp. capitata & $3(88 \%)-4(12 \%)$ & curto & grande & 0,88 & conspícuas \\
B. latifolia & $3(13 \%)-4(85 \%)-5(2 \%)$ & longo & pequena & 0,93 & menos conspícuas \\
& & & & nos pólos; & conspícuas no \\
& & & & & mesocolpo \\
B. uniflora & 3 & muito longo muito pequena 0,85 & pouco conspícuas \\
\hline
\end{tabular}

Tabela 4. Média aritmética (em $\mu \mathrm{m}$ ) das medidas das aberturas e das camadas da exina dos grãos de pólen de espécies de Brunfelsia $(\mathrm{n}=10)$.

\begin{tabular}{|c|c|c|c|c|c|c|c|c|}
\hline \multirow[t]{2}{*}{ Táxons } & \multicolumn{2}{|c|}{ Colpo } & \multicolumn{2}{|c|}{ Endoabertura } & \multirow[t]{2}{*}{ Exina } & \multirow[t]{2}{*}{ Nexina } & \multirow[t]{2}{*}{ Sexina } & \multirow[t]{2}{*}{ Teto } \\
\hline & compr. & larg. & compr. & larg. & & & & \\
\hline B. bonodora & 18,7 & 6,2 & 7,5 & 10,0 & 2,5 & 1,0 & 1,5 & - \\
\hline B. brasiliensis var. brasiliensis & 27,5 & 4,4 & 8,8 & 5,0 & 1,6 & 0,5 & 1,1 & 0,2 \\
\hline B. brasiliensis subsp. macrocalyx & 29,8 & 4,8 & 5,0 & 4,0 & 3,0 & 1,1 & 1,9 & - \\
\hline B. hydrangeiformis subsp. capitata & 22,7 & 4,8 & 4,5 & 3,0 & 2,5 & - & - & - \\
\hline B. latifolia & 24,0 & 5,0 & 7,5 & 5,2 & 2,0 & 0,6 & 1,4 & 0,4 \\
\hline B. uniflora & 32,0 & 5,0 & 5,0 & 11,3 & 2,0 & 1,0 & 1,0 & 0,1 \\
\hline
\end{tabular}

Tabela 5. Média aritmética $($ em $\mu \mathrm{m})$ dos grãos de pólen dos materiais de comparação de espécies de Brunfelsia $(\mathrm{n}=10)$. DP= diâmetro polar; $\mathrm{DE}=$ diâmetro equatorial; $\mathrm{P} / \mathrm{E}=$ relação entre a média do diâmetro polar $(\mathrm{P})$ e a média do diâmetro equatorial $(\mathrm{E})$.

\begin{tabular}{|c|c|c|c|c|}
\hline Táxons & DP & $\mathrm{DE}$ & $\mathrm{P} / \mathrm{E}$ & Forma \\
\hline \multicolumn{5}{|l|}{ B. brasiliensis var. brasiliensis } \\
\hline O.C. Góes e D. Constantino 543 & 46,0 & 47,5 & 0,97 & oblato-esferoidal \\
\hline A. C. Brade 10.583 & 40,5 & 43,0 & 0,94 & oblato-esferoidal \\
\hline \multicolumn{5}{|l|}{ B. brasiliensis subsp. macrocalyx } \\
\hline P.J.M. Maas e G. Martinelli 3.209 & 44,9 & 46,6 & 0,96 & oblato-esferoidal \\
\hline D. Sucre 2.326 e P.I.S. Braga 169 & 49,0 & 53,0 & 0,92 & oblato-esferoidal \\
\hline \multicolumn{5}{|l|}{ B. latifolia } \\
\hline D. Araújo 7.372 & 45,1 & 51,5 & 0,88 & oblato-esferoidal \\
\hline V. Esteves e R. Esteves 910 & 43,5 & 48,2 & 0,90 & oblato-esferoidal \\
\hline \multicolumn{5}{|l|}{ B. uniflora } \\
\hline R. Andreata 100 e H.C. Lima 1.637 & 46,1 & 44,0 & 1,05 & prolato-esferoidal \\
\hline$R B 75.565$ & 45,6 & 43,0 & 1,06 & prolato-esferoidal \\
\hline
\end{tabular}

Os valores do intervalo de confiança a $95 \%$ do diâmetro polar não se interpenetram em todos os táxons estudados porém, quando são analisados os do diâmetro equatorial, apenas em B. hydrangeiformis subsp. capitata e em B. uniflora estes valores são distintos dos demais (tabela 1).

\section{Discussão}

Brunfelsia é um gênero pouco estudado palinologicamente (Rao \& Leong 1974, Miranda et al. 1978, Gentry Jr. 1979, Batista 1999), talvez pela fragilidade dos grãos de pólen ao tratamento acetolítico 
(cinco dos seis táxons estudados foram tratados pela acetólise láctica).

Para os táxons analisados, o número de aberturas, o tipo de endoabertura, a ornamentação da exina e os valores do intervalo de confiança do diâmetro polar foram caracteres importantes na avaliação de cada táxon. Na bibliografia consultada não consta observação de variação no número de aberturas dos grãos de pólen de uma mesma espécie.

Rao \& Leong (1974) e Gentry Jr. (1979) usando o método acetolítico de Erdtman (1952), descreveram os grãos de pólen de $B$. undulata Sw. como possuindo 3poros e exina foveolada. Rao \& Leong (1974) examinaram neste mesmo trabalho os grãos de pólen de $B$. calycina Benth. definindo-os com 4-zonoporados, com exina reticulada. Gentry Jr. (1979) analisou os grãos de pólen de $B$. dwyeri D'Arcy descrevendo-os como colporados, rugulado-foveolados mais do que foveolados. Apesar de não serem espécies aqui analisadas, é importante verificar que os grãos de pólen de Brunfelsia podem apresentar abertura do tipo poro, além do cólporo descrito nos táxons aqui analisados; a ornamentação da exina também varia quando se compara os dados bibliográficos com os do presente estudo (superfície rugulada).

Miranda et al. (1978) descreveram os grãos de pólen de $B$. uniflora como tendo colpos, exina "finamente estriada, com estrias mais densas em direção aos colpos onde a sexina se apresenta granulosa". Os resultados aqui encontrados foram distintos daqueles de Miranda et al. (1978), principalmente no que se refere à ornamentação da sexina e no tipo de abertura (colporado).

Batista (1999) observou que os grãos de pólen de Brunfelsia e Sessea Ruiz \& Pavon (S. brasiliensis Toledo e $S$. regnellii Taub.) apresentavam características próximas, quando foram considerados o tamanho e a forma porém, diferiam na ornamentação da exina (psilada ou rugulada com regiões psiladas no mesocolpo, em Sessea), no número e tipo de aberturas (3-colporados ou 4-loxocolporados em Sessea).

Não foram encontrados dados na literatura para os demais táxons brasileiros de Brunfelsia, podendo-se concluir, assim, que as espécies deste gênero foram muito pouco estudadas palinologicamente; que os táxons estudados até o presente apresentaram certa heterogeneidade, quanto ao número de abertura e quanto à ornamentação da exina, sendo necessários, contudo, estudos futuros para uma visão mais completa da palinologia de Brunfelsia oferecendo, deste modo, mais um auxílio à taxonomia da família.

Agradecimentos - Ao Laboratório de Microscopia Eletrônica do Departamento de Metalurgia da PUC-Rio, na pessoa da técnica MSc. Maria de Fátima Lopes, por fornecer condições de trabalho para a obtenção de eletromicrografias. Ao CNPq pela bolsa de mestrado concedida a primeira autora; à FAPERJ e à FUJB pelos auxílios concedidos ao Laboratório de Palinologia.

\section{Referências bibliográficas}

BARTH, O.M. \& MELHEM, T.S. 1988. Glossário ilustrado de palinologia. Editora da Universidade Estadual de Campinas, Campinas.

BATISTA, C.P.R. 1999. Palinotaxonomia de espécies de Cestroideae Schlecht. (Solanaceae Juss.) ocorrentes no Estado do Rio de Janeiro. Dissertação de mestrado, Museu Nacional/Universidade Federal do Rio de Janeiro, Rio de Janeiro.

CARVALHO, L.D.F. 1997. Diversidade taxonômica das Solanáceas no Estado do Rio de Janeiro (Brasil) - I. Albertoa 4:245-260.

D'ARCY, W.G. 1991. The Solanaceae since 1976, with a review of its biogeography. In Solanaceae III: taxonomy, chemistry, evolution (J.G. Hawkes, R.N. Lester, M. Nee \& N. Estrada, eds.), Royal Botanic Garden Kew and Linnean Society of London, London, p.75-135.

ERDTMAN, G. 1952. Pollen morphology and plant taxonomy - Angiosperms. Almqvist \& Wiksell, Stockholm.

GENTRY Jr., J.L. 1979. Pollen morphology of the Salpiglossideae (Solanaceae). In The biology and taxonomy of the Solanaceae (J.G. Hawkes, R.N. Lester \& A.D. Skelding, eds.), Linnean Society Symposium Séries, London, v. 7, p.327-334.

HOLMGREN, P.K., HOLMGREN, N.H. \& BANETT, L.C. 1990. Index herbariorum. Part 1: The herbaria of the world. $8^{\text {th }}$ ed. New York Botanical Garden, New York.

MIRANDA, M.M.B., PIRES CAVALCANTE, M.P. \& REBOUÇAS GONDIM, M.E. 1978. Pólen das plantas silvestres do Ceará. III. Famílias Cochlospermaceae, Compositae, Dilleniaceae, Melastomataceae, Opiliaceae, Polygalaceae, Solanaceae e Verbenaceae. Revista Brasileira de Farmácia 59:96-104.

PUNT, W., BLACKMORE, S., NILSSON, S. \& LE THOMAS, A. 1999. Glossary of pollen and spore terminology. http://www.biol.ruu.nl/ palaeo/glossary/ glos-int.htm

RAYNAL, J. \& RAYNAL, A. 1971. Une technique de preparation des grains de pollen fragiles. Andansonia 11:77-79. 
RAO, A.N. \& LEONG, F.L. 1974. Pollen morphology of certain tropical plants. Reinwardtia 9:153-176.

REITSMA, T. 1969. Size modification of recent pollen grains under different treatments. Review of Palaeobotany and Palynology 9:175-202.

SALGADO-LABORIAU, M.L. 1973. Contribuição à palinologia dos cerrados. Academia Brasileira de Ciências, Rio de Janeiro.

VIEIRA, S. 1981. Introdução à bioestatística. Editora Campus Ltda, Rio de Janeiro.
WANDERLEY, M.G.L. \& MELHEM, T.S. 1991. Flora Polínica da Reserva do Parque Estadual das Fontes do Ipiranga (São Paulo, Brasil). Família: 178 Bromeliaceae. Hoehnea 18:5-42.

WIEBKE, M.L. \& WIEBKE, G. 1974. Estudo dos grãos de pólen de Solanaceae - 1. Estudos Leopoldinenses 29:71-89.

WODEHOUSE, R.P. 1935. Pollen grains. Their structure, identification and significance in science and medicine. McGraw-Hill Book Company, New York. 